\title{
基于基因组挖掘的一个新的吡嗪酮类天然产物的发现
}

\author{
元文佳 ${ }^{a}$ 吴 晟 ${ }^{b}$ 华会明 $*, a$ 潘海学 ${ }^{b}$ 唐功利 $*, b$ \\ ( ${ }^{a}$ 沈阳药科大学中药学院 基于靶点的药物设计与研究教育部重点实验室 沈阳 110016) \\ ( ${ }^{b}$ 中国科学院上海有机化学研究所 上海 200032)
}

\begin{abstract}
摘要 采用次级代谢产物基因组挖掘的方法, 扫描链霉菌 Streptomyces sp. TP-A0365 的基因组序列, 找到一个新的负责 吡嗪酮类天然产物生物合成的非核糖体肽合成酶(NRPS)编码基因. 通过敲除该 NRPS 基因中断相关化合物的生物合成 途径, 对比突变菌株和原始菌株(来源于链霉菌 Streptomyces sp. TP-A0365 的工程菌株 TG1301)的发酵产物, 我们在原 始菌株的发酵粗提液中捕捉到了突变株不再产生的化合物 1. 从原始菌株 $20 \mathrm{~L}$ 发酵液中分离并鉴定了化合物 1 的化学 结构, 其结构为 3-异丙基-7,8-二氢吡咯并[1,2- $a$ ]吡嗪-4(6H)-酮, 是一个新的吡嗪酮类化合物, 命名为 provalin. 关键词＼cjkstart吡嗪酮; 链霉菌; 基因组挖掘; 生物合成
\end{abstract}

\section{Discovery of a New Pyrazinone Natural Product by Genome Mining}

\author{
Kang, Wen-Jia ${ }^{a} \quad$ Wu, Sheng ${ }^{b} \quad$ Hua, Huiming ${ }^{*, a} \quad$ Pan, Hai-Xue ${ }^{a} \quad$ Tang, Gong-Li ${ }^{*, b}$ \\ $\left({ }^{a}\right.$ Key Laboratory of Structure-Based Drug Design \& Discovery, Ministry of Education, Shenyang \\ Pharmaceutical University, Shenyang 110016) \\ ( ${ }^{b}$ Shanghai Institute of Organic Chemistry, Chinese Academy of Sciences, Shanghai 200032)
}

\begin{abstract}
The genomic sequences of Streptomyces sp. TP-A0365 were scanned using a secondary metabolite genome mining approach. A new nonribosomal peptide synthetase (NRPS) gene responsible for the biosynthesis of an unknown pyrazinone compound was found. The biosynthetic pathway of related compound was disrupted by inactivating the NRPS gene. Comparing the fermentation products of the mutant strain and the original strain (TG1301 derived from Streptomyces sp. TP-A0365), we caught compound 1 which disappeared in the mutant strain. Compound 1 was isolated from $20 \mathrm{~L}$ fermentation cultures of the original strain and its structure was established as 3-isopropyl-7,8-dihydropyrrolo[1,2- $a$ ]pyrazin-4(6H)-one, a new pyrazinone compound designated as provalin
\end{abstract}

Keywords pyrazinone; Streptomyces; genome mining; biosynthesis

吡嗪酮(pyrazinone)类天然产物是由微生物产生的 含非芳香吡嗪酮环的化合物, 目前已发现的这类天然产 物包括：耐甲氧西林金黄色葡萄球菌(MRSA)产生的 tyrvalin, phevalin 和 leuvalin ${ }^{[1,2]}$, 链霉菌产生的 phevalin, arglecin, argvalin, maremycin E/F, JBIR-56/-57 $7^{[3 \sim 6]}$, 粘细 菌产生的 nannozinone $\mathrm{A} / \mathrm{B}$ 和 sorazinone $\mathrm{B}^{[7]}$, 疮狍菌产 生的 butrepyrazinone ${ }^{[8]}$, 以及类芽孢杆菌产生的 paenibacillin $\mathrm{A}^{[9]}$. 吡嗪酮类天然产物的抗菌活性较弱, 研究发现其作为信号分子在 MRSA 等病原菌的感染中 起着关键作用 ${ }^{[1,10]}$; 同时, 一些经化学衍生的吡嗪酮类 化合物是微管蛋白、丙型肝炎 NS3 蛋白酶等靶标的良好 抑制剂 ${ }^{[11-13]}$. 因此, 发现具有新颖骨架结构的吡嗪酮化

\section{合物具有重要意义.}

随着基因测序技术的进步和生物合成研究的深入, 近年来出现了一种全新的天然产物发现模式, 即基于生 物信息指导发现新化合物的方法一一基因组挖掘. 已有 研究表明, 吡嗪酮天然产物的核心骨架由非核糖体肽合 成酶(nonribosomal peptide synthetases, NRPSs)催化形成, 两分子氨基酸通过双模块 NRPS 活化和缩合后，在末端 还原酶结构域(reductase, RE)的作用下还原解离并环 化 ${ }^{[2,14]}$. 本课题组采用基因组扫描的方法寻找可能负责 新吡嗪酮天然产物生物合成的基因, 通过基因操作、对 比发酵、化合物分离和结构鉴定, 从链霉菌 Streptomyces sp. TP-A0365 中发现一个新的吡嗪酮类天然产物

\footnotetext{
*E-mail: huimhua@163.com; gltang@sioc.ac.cn

Received February 18, 2016; revised March 1, 2016; published online March 18, 2016.

Project supported by the National Natural Science Foundation of China (Nos. 81373307 \& 81473124).

国家自然科学基金(Nos. 81373307 \& 81473124)资助项目.
} 
provalin (图 1). 与之前发现的此类化合物的氨基酸组成 不同, provalin 由脯氨酸和烦氨酸缩合并环化形成 $5 \sim 6$ 并环结构. 本研究结果为该类化合物的化学衍生提供了 新的选择, 同时也展示了基因组挖掘在定向发现新天然 产物方面的高效性.

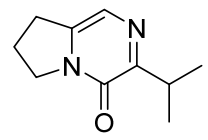

图 1 Provalin (1)结构图

Figure 1 Structure of provalin (1)

\section{1 结果与讨论}

本课题组在分析链霉菌 Streptomyces sp. TP-A0365 的基因组时发现, 其中包含一个两模块 NPRS 的编码基 因, 其结构域组成与已知吡嗪酮类天然产物的 NPRS 相 似, 且蛋白同源性较高, 但是 NRPS 识别的两个氨基酸 底物与已知的该类化合物不同, 推测其编码一个新的吡 嗪酮类天然产物. 由于该链霉菌是天然产物 yatakemycin 的产生菌, 为了排除此类化合物的干扰, 我 们选择敲除了 yatakemycin 生物合成基因簇的菌株 TG1301作为原始菌株 ${ }^{[15]}$, 采用同源重组双交换的方法 敲除 NRPS 基因, 经聚合酶链式反应(PCR)验证获得基 因型正确的突变株命名为 dNRPS(图 2A). 采用原始发 酵条件 ${ }^{[15]}$, 将突变株 dNRPS 与 TG1301 同时发酵. 发酵 粗提液的高效液相色谱 (HPLC) 分析结果显示, 与 TG1301相比, dNRPS 突变株不再产生保留时间 $13.1 \mathrm{~min}$ 的化合物 1 (图 2B). LC-MS 分析显示该化合物 $m / z 179.1$ $[\mathrm{M}+\mathrm{H}]^{+}$与推测的脯氨酸和缬氨酸底物缩合形成的的吡
嗪酮天然产物的分子量相符. 该化合物同时也存在于野 生型链霉菌 Streptomyces sp. TP-A0365 的发酵产物中.

将菌株 TG1301 进行大量发酵 $(20 \mathrm{~L})$, 并通过反向 硅胶及半制备高效液相色谱分离纯化获得了化合物 $\mathbf{1}$. 该化合物为无色固体, 紫外光谱于 $230,320 \mathrm{~nm}$ 有强吸 收, 高分辨质谱(HR-ESIMS)给出其准分子离子峰 $\mathrm{m} / \mathrm{z}$ $179.1177[\mathrm{M}+\mathrm{H}]^{+}$, 提示其分子式为 $\mathrm{C}_{10} \mathrm{H}_{14} \mathrm{~N}_{2} \mathrm{O}$ (计算值 $\left.179.1184[\mathrm{M}+\mathrm{H}]^{+}\right)$.

化合物 1 的 ${ }^{1} \mathrm{H}$ NMR 谱(溶剂是 DMSO- $d_{6}$ ) 存在两个 甲基氢信号 $\left(\delta_{\mathrm{H}} 1.10,1.12\right)$, 三个亚甲基氢信号 $\left(\delta_{\mathrm{H}} 2.10\right.$, $3.04,3.98)$, 一个脂肪链次甲基氢信号 $\left(\delta_{\mathrm{H}} 3.28\right)$, 一个烯 烃氢信号 $\left(\delta_{\mathrm{H}} 7.25\right) .{ }^{13} \mathrm{C}$ NMR 谱显示有 10 个碳信号: 结 合 DEPT 谱能指认其中两个甲基碳信号 $\left(\delta_{\mathrm{H}} 20.2,20.2\right.$, 重叠); 三个仲碳信号 $\left(\delta_{\mathrm{H}} 21.0,28.8,48.5\right)$; 两个叔碳信 号, 其中一个为烯烃碳 $\left(\delta_{\mathrm{H}} 117.2\right)$, 另一个为脂肪链次甲 基碳 $\left(\delta_{\mathrm{H}} 29.3\right)$; 三个季碳 $\left(\delta_{\mathrm{H}} 141.4,154.3,158.6\right)$ (表 1). 通过 HSQC 谱, 我们能确定相关的碳信号对应的氢信 号, ${ }^{1} \mathrm{H}-{ }^{1} \mathrm{H}$ COSY 谱中, $\mathrm{H}-5$ 分别与 $\mathrm{H}-1, \mathrm{H}-2 ; \mathrm{H}-3$ 分别与 H-4、H-6 有氢氢相关. HMBC 谱中, H-1, H-2, H-5 都与 C-10 相关, 由此能确定这一部分连接在 C-10 位. 另外 $\mathrm{H}-3$ 与 C-4, C-6, C-8; H-4 与 C-3, C-6, C-7, C-8; H-6 与 C-3, C-4, C-8, C-9 都有相关信号, 由此能确定这部分吡 咯环结构并且得出氮原子连接着羰基. 烯烃的氢原子 H-7 与 C-4, C-8, C-10 也有远程相关, 更加确证了这一结 构(图 3). 根据以上信息, 化合物 $\mathbf{1}$ 的结构鉴定为: 3-异 丙基-7,8-二氢吡咯并 $[1,2-a]$ 吡嗪-4(6H)-酮, 命名为 provalin (图 1).
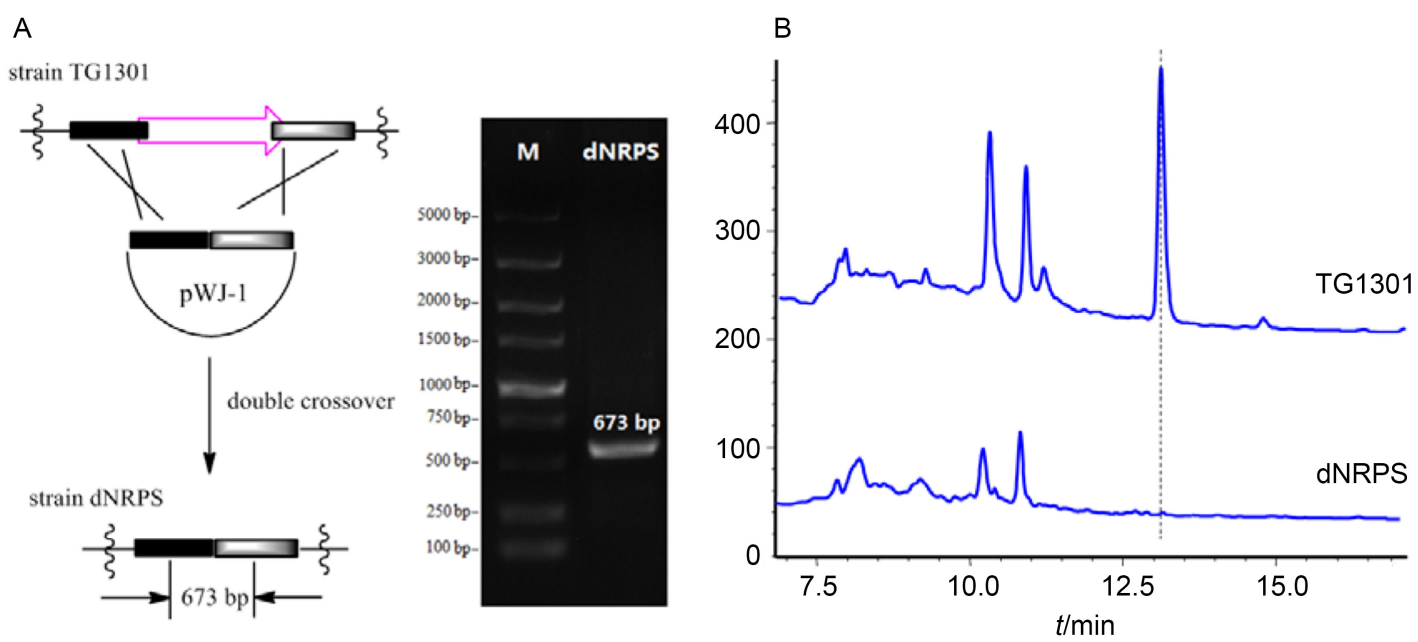

图 2 (A)突变株构建和验证以及(B)菌株 TG1301 和突变株 dNRPS 的发酵粗提物 HPLC 分析

Figure 2 (A) Construction and verification of mutant strain, and (B) HPLC analysis of fermentation extractions of strains TG1301 and dNRPS 
表 1 化合物 1 核磁共振图谱数据(500/125 MHz, DMSO- $d_{6}$ )

Table 1 NMR spectroscopic data of compound 1 (500/125 MHz, DMSO- $d_{6}$ )

\begin{tabular}{clllc}
\hline Position & \multicolumn{1}{c}{$\delta_{\mathrm{C}}$} & \multicolumn{1}{c}{$\delta_{\mathrm{H}}$} & $\mathrm{HMBC}(\mathrm{H} \rightarrow \mathrm{C})$ & ${ }^{1} \mathrm{H}-{ }^{1} \mathrm{H} \mathrm{COSY}$ \\
\hline 1 & $20.2 \mathrm{q}$ & $1.10(\mathrm{~s}, 3 \mathrm{H})$ & $\mathrm{C}-5,10$ & $\mathrm{H}-5$ \\
2 & $20.2 \mathrm{q}$ (重叠) & $1.12(\mathrm{~s}, 3 \mathrm{H})$ & $\mathrm{C}-5,10$ & $\mathrm{H}-5$ \\
3 & $21.0 \mathrm{t}$ & $2.10(\mathrm{~m}, J=7.5 \mathrm{~Hz}, 2 \mathrm{H})$ & $\mathrm{C}-4,6,8$ & $\mathrm{H}-4,6$ \\
4 & $28.8 \mathrm{t}$ & $3.04(\mathrm{t}, J=7.5 \mathrm{~Hz}, 2 \mathrm{H})$ & $\mathrm{C}-3,6,7,8$ & $\mathrm{H}-3$ \\
5 & $29.3 \mathrm{~d}$ & $3.28(\mathrm{~m}, J=7.0 \mathrm{~Hz}, 1 \mathrm{H})$ & $\mathrm{C}-1,2,9,10$ & $\mathrm{H}-1,2$ \\
6 & $48.5 \mathrm{t}$ & $3.98(\mathrm{t}, J=7.5 \mathrm{~Hz}, 2 \mathrm{H})$ & $\mathrm{C}-3,4,8,9$ & \\
7 & $117.2 \mathrm{~d}$ & $7.25(\mathrm{~s}, 1 \mathrm{H})$ & $\mathrm{C}-4,8,10$ & \\
8 & $141.4 \mathrm{~s}$ & & & \\
9 & $154.3 \mathrm{~s}$ & & & \\
10 & $158.6 \mathrm{~s}$ & & & \\
\hline
\end{tabular}

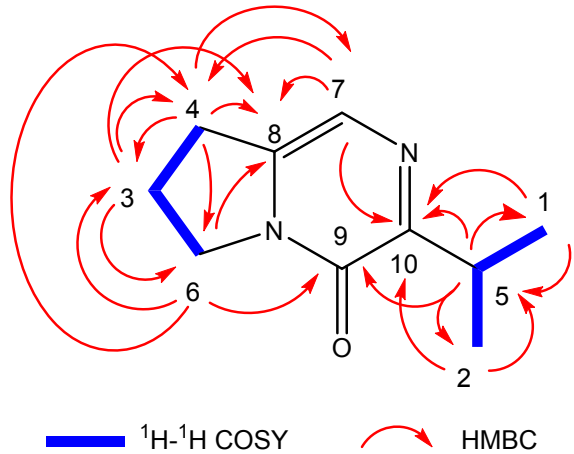

图 3 化合物 $\mathbf{1}$ 的 $\mathrm{HMBC}$ 与 ${ }^{1} \mathrm{H}-{ }^{1} \mathrm{H}$ COSY 相关 Figure $3 \mathrm{HMBC}$ and ${ }^{1} \mathrm{H}_{-}{ }^{1} \mathrm{H} \mathrm{COSY}$ correlations of compound 1

\section{2 实验部分}

\section{1 仪器与试剂、生物材料}

PCR 扩增仪 Eppendorf Mastercycler, 凝胶成像系统 BioRad Syngene, 质谱仪 Thermo Scientific LCQ Fleet Ion Trap, 高效液相色谱仪 Agilent 1260, 高分辨质谱仪 Agilent Technologies 6230 TOF LC/MS, 核磁共振仪 Bruker AVANCE DRX-500, 分析色谱柱 Diamonsil C18 $5 \mu 250 \mathrm{~mm} \times 4.6 \mathrm{~mm}$, 半制备柱 Venusil XBP C18, $5 \mu \mathrm{m}$, $100 \AA, 250 \mathrm{~mm} \times 10 \mathrm{~mm}$. 生物试剂购自 Takara 公司, 引 物合成和 DNA 测序由苏州金唯智公司完成, 进口化学 试剂购自 Sigma-Aldrich 公司, 国产化学试剂购自上海 国药. 链霉菌 Streptomyces sp. TP-A0365 由日本富山县 立大学的 Yasuhiro Igarashi 教授提供, 菌株 TG1301 由本 实验室构建, 其它大肠杆菌和质粒由本实验室保存.

\section{2 构建 NRPS 基因敲除突变株}

在 $\mathrm{pKC} 1139$ 载体中构建含左右臂的同框敲除质粒 pWJ-1, 左臂引物为 PYR-L1 (GAATTCCAACTGCTGCTCGGCACCTG)和 PYR-L2 (TCTAGAGCCGTAGCCGAGTCGAACGTC), 右臂引物为 PYR-R1 (TCTAGAGACGTACCTGCCGGAAGAGTG) 和 PYR-R2 (AA-
GCTTCTGACCGAGTTCTTCACCAC). 同框敲除质粒 构建、接合转移及突变株笁选方法参考文献 ${ }^{[15]}$, 通过 PCR 验证突变株的基因型，验证引物为 PYR-V1 (CACTCACAGGAAGGCAGGA) 和 PYR-V2 (TCGTTGCGCAGATGTCCGGAG), 目标条带大小为 673 bp, 获 得正确的 NRPS 基因同框敲除突变株 dNRPS.

\section{3 突变株的发酵和化合物 1 的提取和分离}

菌株 TG1301 和 dNRPS 采用文献中的方法发酵 ${ }^{[15]}$, 收集 $50 \mathrm{~mL}$ 发酵液菌体用 3 倍体积丙酮浸泡并超声 10 $\min$, 减压浓缩至干, 用 $0.5 \mathrm{~mL}$ DMSO 溶解, 样品离心 去沉淀后供 HPLC 和 LC-MS 分析. 收集 $20 \mathrm{~L}$ 菌株 TG1301 的发酵液菌体，用丙酮提取 2 次，离心去沉淀， 上清旋转蒸发除去丙酮, 剩余水相用乙酸乙酯萃取 2 次, 有机相减压浓缩得浸膏，用 DMSO 溶解浸膏，经反向硅 胶柱初步分离纯化, 半制备高效液相色谱进一步纯化 $[V$ (水) $: V$ (乙腈 $)=7: 3 \sim 2: 8$, 流速 $3 \mathrm{~mL} / \mathrm{min}$, 检测波 长 $320 \mathrm{~nm}$, 最终得化合物 1 (15 mg).

\section{4 发酵结果分析与化合物 1 结构鉴定}

HPLC 分析：将菌株 dNRPS 与 TG1301 的发酵粗提 液分别用 HPLC 和 LC-MS 检测, 通过对比出峰情况确 定 NRPS 基因敲除后菌株发酵产物的变化, 以此确定 TG1301 发酵产物中的目标化合物. HPLC 条件: A 相为 水(含 $0.1 \%$ 甲酸), $\mathrm{B}$ 相为乙腈(含 $0.1 \%$ 甲酸), 流速 1 $\mathrm{mL} / \mathrm{min}$, 检测波长 $320 \mathrm{~nm}$ 及全波长扫描，洗脱梯度 0 $3 \mathrm{~min}, 10 \% \mathrm{~B} ; 3 \sim 10 \mathrm{~min}, 10 \% \sim 40 \% \mathrm{~B} ; 10 \sim 21 \mathrm{~min}$, $40 \% \sim 80 \% \mathrm{~B} ; 21 \sim 25 \mathrm{~min}, 80 \% \sim 10 \% \mathrm{~B}$. LC-MS 采用与 HPLC 相同的条件. 对于纯化得到的化合物 $\mathbf{1}$, 进行高分 辨质谱和 NMR 分析，得到化合物完整的结构信息.

致谢 感谢日本富山县立大学的 Yasuhiro Igarashi 教授 提供 Streptomyces sp. TP-A0365 菌株. 
辅助材料(Supporting Information) 化合物 1 的紫外吸 收光谱, MS, HR-ESIMS, NMR 图谱. 这些材料可以免费 从本刊网站(http://sioc-journal.cn/)上下载.

\section{References}

[1] Wyatt, M. A.; Wang, W.; Roux, C. M.; Beasley, F. C.; Heinrichs, D. E.; Dunman, P. M.; Magarvey, N. A. Science 2010, 329, 294.

[2] Zimmermann, M.; Fischbach, M. A. Chem. Biol. 2010, 17, 925.

[3] Alvarez, M. E.; White, C. B.; Gregory, J.; Kydd, G. C.; Harris, A.; Sun, H. H.; Gillum, A. M.; Cooper, R. J. Antibiot. 1995, 48, 1165.

[4] Tatsuta, K.; Fujimoto, K.; Yamashita, M.; Tsuchiya, T.; Umezawa, S. J. Antibiot. 1973, 26, 606

[5] Tang, Y.-Q.; Sattler, I.; Thiericke, R.; Grabley, S.; Feng, X.-Z. Eur. J. Org. Chem. 2000, 2, 261.

[6] Motohashi, K.; Inaba, K.; Fuse, S.; Doi, T.; Izumikawa, M.; Khan, S. T.; Takagi, M.; Takahashi, T.; Shinya, K. J. Nat. Prod. 2011, 74, 1630.

[7] Jansen, R.; Sood, S.; Mohr, K. I.; Kunze, B.; Irschik, H.; Stadler, M.; Müller, R. J. Nat. Prod. 2014, 77, 2545.

[8] Kyeremeh, K.; Acquah, K. S.; Camas, M.; Tabudravu, J.; Houssen, W.; Deng, H.; Jaspars, M. Mar. Drugs 2014, 12, 5197.
[9] Bian, X.; Shao, M.; Pan, H.; Wang, K.; Huang, S.; Wu, X.; Xue, C.; Hua, H.; Pei, Y.; Bai, J. Nat. Prod. Res. 2016, 30, 125.

[10] Secor, P. R.; Jennings, L. K.; James, G. A.; Kirker, K. R.; Pulcini, E. D.; McInnerney, K.; Gerlach, R.; Livinghouse, T.; Hilmer, J. K.; Bothner, B.; Fleckman, P.; Olerud, J. E.; Stewart, P. S. PLoS One 2012, 7, e40973.

[11] Taggi, A. E.; Stevenson, T. M.; Bereznak, J. F.; Sharpe, P. L.; Gutteridge, S.; Forman, R.; Bisaha, J. J.; Cordova, D.; Crompton, M.; Geist, L.; Kovacs, P.; Marshall, E.; Sheth, R.; Stavis, C.; Tseng, C. P. Bioorg. Med. Chem. 2016, 24, 435.

[12] Gising, J.; Belfrage, A. K.; Alogheli, H.; Ehrenberg, A.; Åkerblom, E.; Svensson, R.; Artursson, P.; Karlén, A.; Danielson, U. H.; Larhed, M.; Sandström, A. J. Med. Chem. 2014, 57, 1790.

[13] Zhang, X.; Glunz, P. W.; Jiang, W.; Schmitt, A.; Newman, M.; Barbera, F. A.; Bozarth, J. M.; Rendina, A. R.; Wei, A.; Wen, X.; Rossi, K. A.; Luettgen, J. M.; Wong, P. C.; Knabb, R. M.; Wexler, R. R.; Scott Priestley, E. Bioorg. Med. Chem. Lett. 2013, 23, 1604.

[14] Wyatt, M. A.; Mok, M. C.; Junop, M.; Magarvey, N. A. Chembiochem 2012, 13, 2408.

[15] Huang, W.; Xu, H.; Li, Y.; Zhang, F.; Chen, X.-Y.; He, Q.-L.; Igarashi, Y.; Tang, G.-L. J. Am. Chem. Soc. 2012, 134, 8831. 